\title{
Extraction on Paper, an Active Learning Technique to Facilitate Student Understanding of Liquid-Liquid Extraction
}

Kate J. McKnelly, William J. Howitz, Simon Lam, Renée D. Link*

Department of Chemistry, University of California-Irvine, Irvine, CA 92697, United

5 States

\begin{abstract}
Undergraduate students often find it difficult to understand the chemical principles underlying liquid-liquid extractions. Explanations on how extractions work at the molecular level in textbook and internet resources are plentiful, but students still do not seem able to grasp how extractions work before having to perform the technique in a laboratory course. To address this difficulty in understanding, we developed an Extraction on Paper Activity. We envision this activity as a tool to help students understand and apply the chemical principles underlying liquid-liquid extractions outside of and before entering into a laboratory setting.
\end{abstract}


Extraction Activity: Please fill in all blanks (indicated with ?)

Top Layer Solvent:

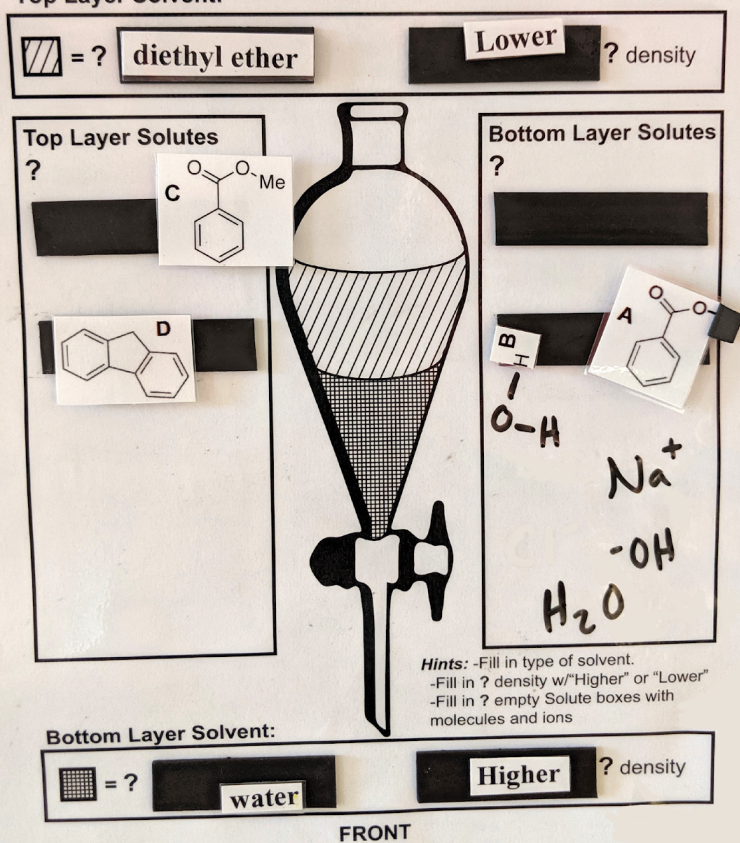

\section{KEYWORDS}

High School, Introductory Chemistry, First-Year Undergraduate, General Chemistry, Second-Year Undergraduate, Organic Chemistry, Laboratory Instruction, Hands-On

\section{INTRODUCTION}

Liquid-liquid extraction is a ubiquitous technique taught in undergraduate chemistry laboratory courses. Undergraduate students have resources in the form of lectures, textbooks, video podcasts, lab manuals, live demos, etc. to help them understand and apply the chemical principles behind liquid-liquid extraction. Despite the abundance of available resources, many students continue to struggle with connecting the conceptual aspects of how liquid-liquid extractions, including acid- 
base extractions, work at the molecular level to the physical process of extraction. We have developed an active learning technique in the form of an Extraction on Paper Activity to aid student understanding of liquid-liquid extractions. This activity has been used in large lecture settings, and can be used in any classroom or laboratory setting.

Active learning proves to be an effective learning technique that engages students and helps them better conceptualize difficult material. Many researchers in science, technology, engineering, and mathematics (STEM) education disciplines have demonstrated that incorporating active learning in lecture and discussion settings greatly improves student performance on challenging course material. ${ }^{1}$ Active learning can take many forms, from think-pair-share and group work to clicker questions and physical activities that students can manipulate.

Chemistry educators have often turned to hands-on active learning techniques that allow students to interact with a chemistry lab technique in a physical or virtual form. ${ }^{2-6}$ The goal of these active learning techniques is to help students perform inlab experiments with greater comfort and expertise.

Two more recent active learning techniques, involving dyes, were developed as guided laboratory extraction activities. ${ }^{7,8}$ The activities were meant to help students understand extraction at the molecular level, including principles of immiscibility, solubility, acid-base reactions in the context of extraction, mixture equilibrium, and planning how to execute an extraction. While these activities are useful and effective in a laboratory setting, they are not amenable to lectures, discussions, and other 
learning environments outside of a lab due to the use of hazardous chemicals that would not be appropriate in these settings.

Hill and McGurran developed a model to help students visualize the molecular interactions that occur in a liquid-liquid extraction, particularly how polar molecules interact with the more polar solvent and how nonpolar molecules interact with the more nonpolar solvent. 9 They used cardboard covered with two different pieces of colored paper to represent two immiscible layers, and Styrofoam balls matching each color of the "layers" to represent polar and nonpolar molecules. Hill and McGurran's activity was tactile and allowed students to attach the molecules with different polarities to their respective layers using Velcro. This simple model only illustrated 60 principles of solubility and miscibility in the context of a liquid-liquid extraction. The Extraction on Paper Activity we present in this paper incorporates principles of polarity and extends the concepts illustrated to include how acid-base extraction works at the molecular level. We also provide a means for students to "separate" the immiscible layers and continue the extraction purification to the point of isolating the desired product.

Our approach to aiding student understanding of liquid-liquid extraction is to provide students with a physical kit that facilitates the performance of a dry extraction on paper. We developed an Extraction on Paper Activity kit containing a laminated worksheet that can be used in any learning environment. The kits are 70 designed so an instructor or teaching assistant can guide a course, or a student can work independently, through the Extraction on Paper Activity. 


\section{EXTRACTION ON PAPER ACTIVITY DESIGN}

The Extraction on Paper Activity kits were designed to provide students with a

tactile learning experience that guides them step-by-step through a liquid-liquid

extraction process. The worksheet depicts an image of a separatory funnel with boxes

labeled as top or bottom layer (Figure 1). Spaces are included for students to label the layers with lower or higher density. The back of the worksheet has general instructions for using the activity and an image of two beakers labeled aqueous layer and organic layer. Once printed, the worksheets were laminated and velcro or

magnets were pasted onto the blank spaces of the worksheet. The corresponding labels were also laminated and affixed with either velcro or magnets. The activity kits were then assembled with the complete worksheet, labels, a dry-erase marker and a felt eraser. Full worksheets and labels are provided in the Supporting Information.
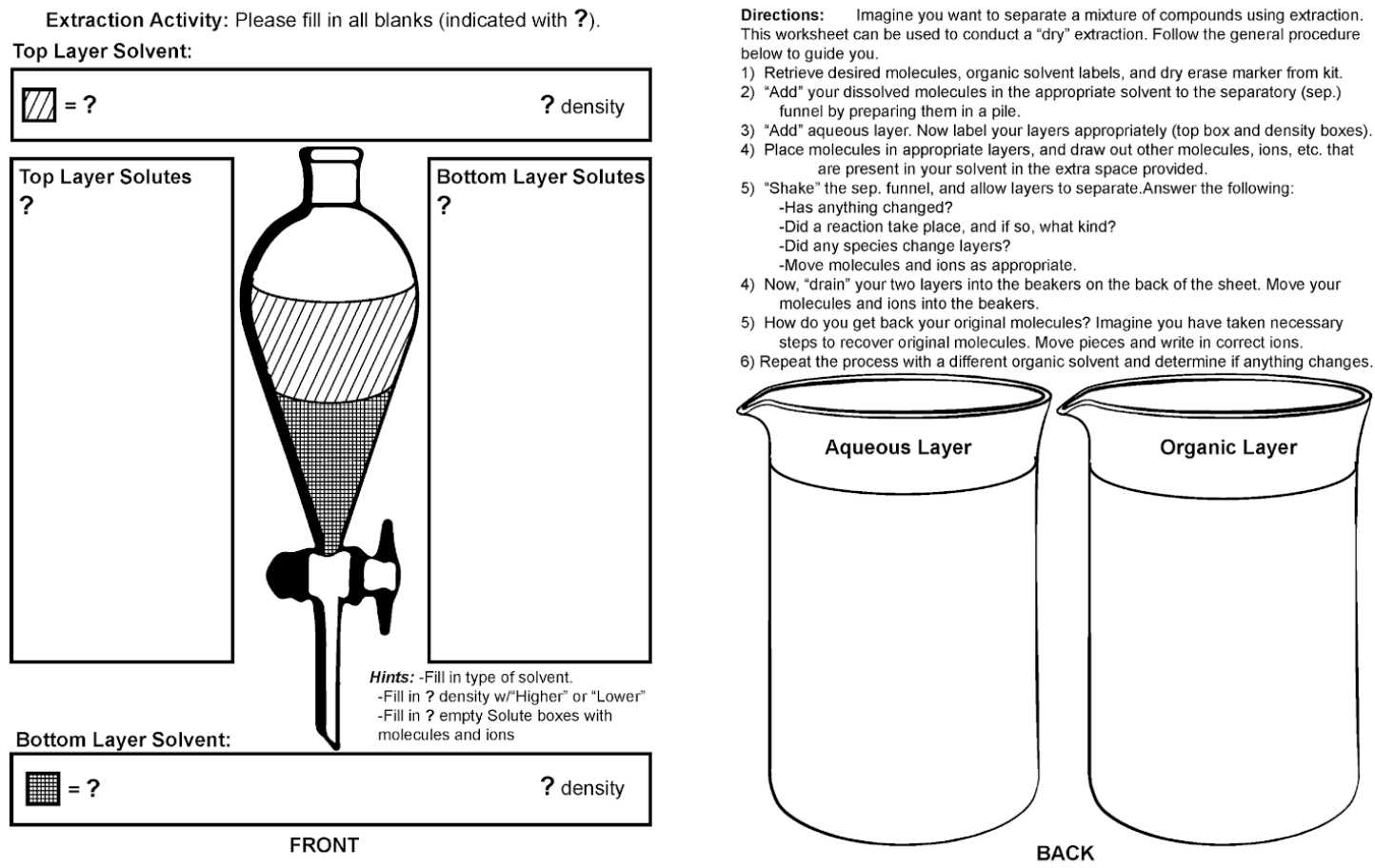
Each part of the Extraction on Paper Activity worksheet was designed to illustrate important concepts and chemical principles underlying liquid-liquid extraction. Labels corresponding to the top and bottom layer solvent identities were included to show that the two solvents chosen for an extraction should be immiscible. Spaces next to the top and bottom layer solvent identities should be filled with either the label "Higher" or the label "Lower" to indicate where the higher or lower density liquids will be located. Students must locate the densities of the two solvents they are using in any appropriate reference material such as a lab manual or SDS file to determine which solvent will be in which layer. The provided dry-erase markers are useful to write the density next to each density label.

Acid-base chemistry is also amenable to demonstration with the Extraction on Paper Activity. (Figure 2) In our example, we have the students imagine they are in a laboratory and they want to separate benzoic acid (A-B) from a compound mixture containing benzoic acid (A-B), methyl benzoate (C) and fluorene (D). The only way to separate benzoic acid from this mixture is to exploit it as an acid. We designed a label with a benzoic acid molecule (A) that has a removable proton (B). After "dissolving" our mixture in an organic solvent such as dichloromethane or diethyl ether, we "add" aqueous sodium hydroxide solution to the separatory funnel on the Extraction on Paper Activity. Before mixing, we tell the students to label both layers and place all molecules in the correct Solutes box. We then instruct the students to "shake" the separatory funnel, and describe what happens. Are the solvents interacting more? Can a chemical reaction now take place? We guide the students through an acid-base reaction between $\mathrm{NaOH}$ and benzoic acid by instructing them to remove the proton 
from the acid and then transferring the charged carboxylate to the aqueous layer

Solutes box (Bottom Layer Solutes in Figure 2).
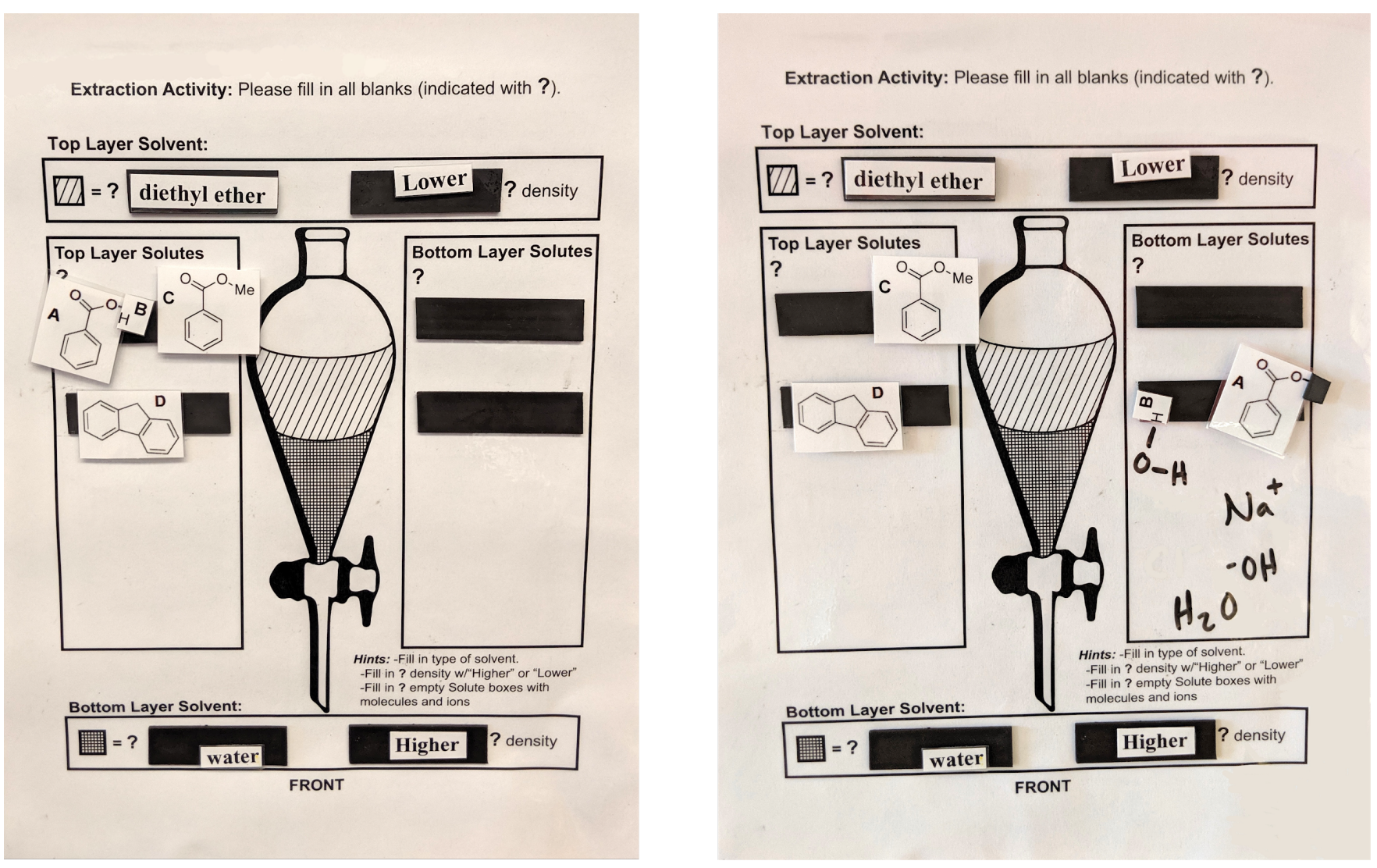

Figure 2. Demonstration of the Extraction on Paper Activity depicting acid-base extraction before and after adding aqueous base.

After completing the "separation" of benzoic acid with the compound mixture, we then ask the students to flip the Extraction on Paper Activity to the BACK side (Figure 1). Students are then instructed to "drain" the layers into the appropriately labeled beakers and transfer all molecules from the FRONT of the worksheet to the BACK beakers. To complete the process of extracting on paper, students must recover the original desired molecule, benzoic acid. Students must "add" aqueous hydrochloric acid solution to re-protonate the carboxylate anion, and recover the benzoic acid through "precipitation." Images of these steps are provided in the 
Supporting Information. This stepwise procedure was designed to help students understand the chemical principles that allow a liquid-liquid extraction to work at the molecular level and connect these principles to each step of extraction they perform in a lab setting.

\section{ACTIVITY IMPLEMENTATION AND ASSESSMENT}

The Extraction on Paper Activity was used in the lab lecture component of the first course in a series of organic chemistry laboratory courses, having as few as 71 students and as many as 442 students in a large lecture hall setting. The activity has been used in the lab lecture component of the same course in three different academic terms, including an accelerated summer term. Because the activity was designed to be used during lab lecture, 200 kits were constructed to allow students to work in groups of two or three.

Two rounds of refinement were undertaken to transform the original activity to the version presented here (Table 1). In the first and second iterations, anecdotal feedback was collected from students and the activity instructions were modified for clarity. Concurrent with the third iteration of the activity, a formal survey was administered to determine students' self-reported comfort levels and understanding of extraction before and after the activity (Table 2).

Table 1. Extraction on Paper Activity Refinement

Iteration 1

1. Designed activity

2. Used activity in lab lecture

3. Collected survey data on what worked well and what didn't work well
Iteration 2

1. Refined activity

2. Used activity in lab lecture

3. Collected survey data on what worked well and what didn't work well

\section{Iteration 3}

4. Refined activity

5. Used activity in lab lecture

6. Collected data to test student understanding of liquid-liquid extraction (presented here) 
Table 2. Survey Questions

\section{Questions}

Q1. BEFORE class, how comfortable would you feel if asked to explain HOW acid-base extraction works at the molecular level?

Q2. BEFORE class, how comfortable would you feel if asked to explain WHY acid-base extraction works at the molecular level?

Q3. AFTER class, how comfortable would you feel if asked to explain HOW acid-base extraction works at the molecular level?

Q4. AFTER class, how comfortable would you feel if asked to explain WHY acid-base extraction works at the molecular level?

The survey administered for the third iteration of the Extraction on Paper Activity consisted of questions on a Likert scale with response choices labeled: (1) extremely uncomfortable, (2) moderately uncomfortable, (3) slightly uncomfortable, (4) neither comfortable nor uncomfortable, (5) slightly comfortable, (6) moderately comfortable, and (7) extremely comfortable. Most students self-reported having greater comfort in their understanding of extraction after the activity. When asked how comfortable students were with understanding how extraction works at the molecular level before the activity, $34 \%$ of students responded (1), (2), (3), or (4), indicating a low level of comfort in their understanding. (Figure 3). Similarly, 38\% of students reported that they were uncomfortable with understanding why extraction works at the molecular level before the Extraction on Paper Activity. To compare student comfort in their understanding before and after the Extraction on Paper Activity, the students were then asked to report how comfortable they were in understanding extraction after the 
activity. After the activity, $77 \%$ of students indicated that they were comfortable in their understanding of how extraction works at the molecular level, and $82 \%$ of students indicated that they were comfortable in their understanding of why extraction works at the molecular level. Sixty-eight percent of students indicated that their comfort level with understanding how and why extraction works increased after completing the Extraction on Paper Activity (Table 3).

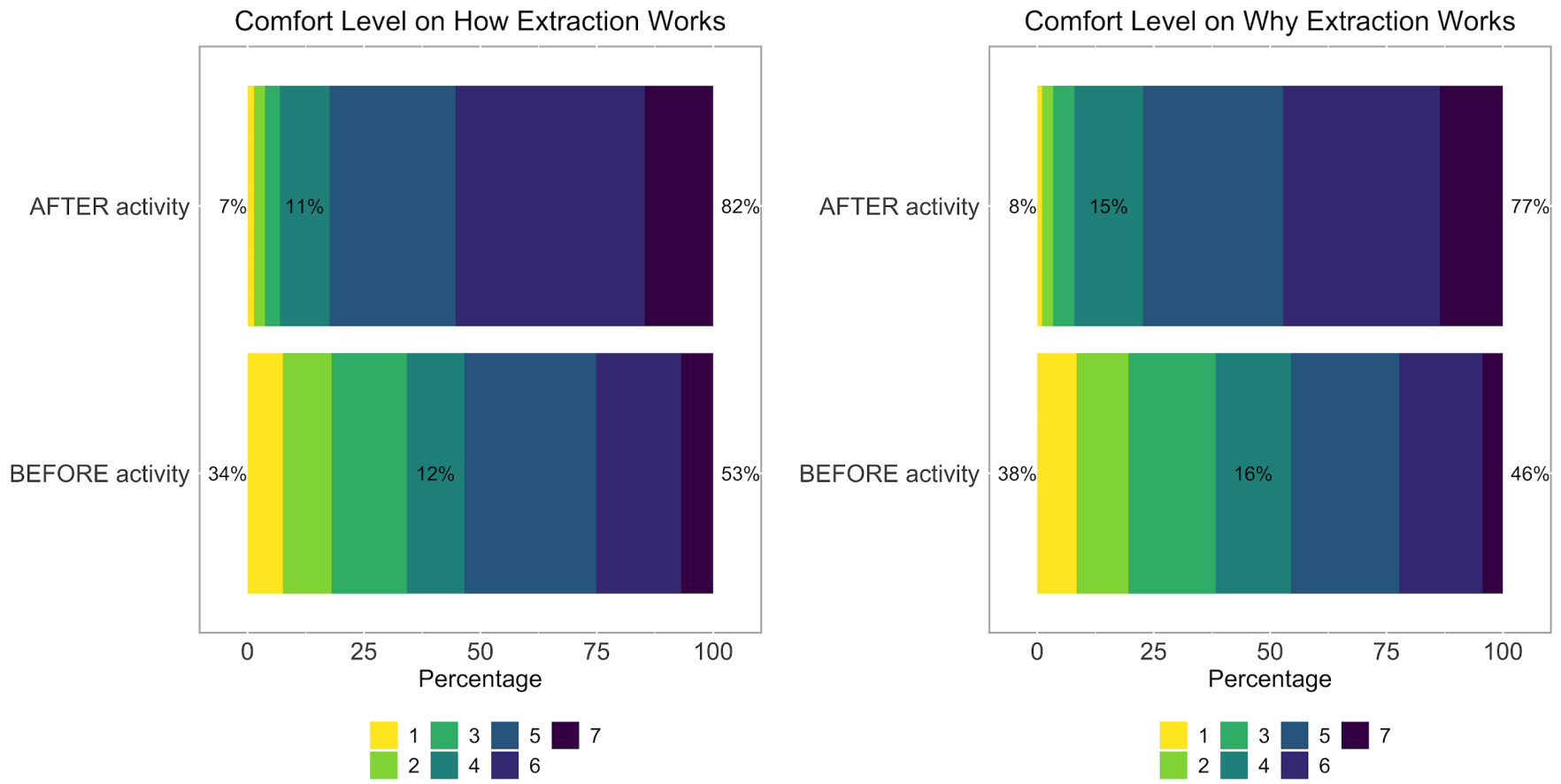

Figure 3. Student Self-Reported Comfort Level Before and After Activity

Table 3. Student Reported Change in Comfort Level with How and Why Extraction Works

$\begin{array}{lcc}\text { Question Pair } & \text { Increase } & \text { No Increase } \\ \text { Q1/Q3 } & 383(64 \%) & 215 \\ \text { Q2/Q4 } & 369(64 \%) & 211 \\ \text { Q1|Q3 \& Q2 |Q4 } & 294(68 \%) & 139 \\ \text { Totals are not equal because not all students answered every } \\ \text { question. These reflect only full answers for each question pair. }\end{array}$


Because the majority of students self-reported that they were more comfortable with their understanding of extraction after the Extraction on Paper Activity, we included a concept question on the formal survey to check their understanding of extraction (Table 4). The students were given four answer choices designed to test their understanding of acid-base reactions and solubility. Although around $68 \%$ of students indicated that they felt more comfortable with extraction after the

Extraction on Paper Activity, nearly half of these students did not answer the concept question correctly (Figure 4). Of the students who reported a decrease or no change in their comfort level in understanding extraction, about half of the students answered the concept question correctly. Time constraints in a one-hour lecture period could account for students not performing as well on this question as they could have if given more time to think about the question.

Table 4. Concept Check for Understanding

\section{Question}

Which pairs of statements is completely true?

\section{Possible Responses}

Adding strong acid to benzoic acid (dissolved in the organic solvent) will cause deprotonation of benzoic acid (now negatively charged). The compound was in the aqueous layer, but after mixing it is in the organic layer.

Adding strong base to benzoic acid (dissolved in the organic solvent) will cause deprotonation of benzoic acid (now negatively charged). The compound was in the aqueous layer, but after mixing it is in the organic layer.

Adding strong base to benzoic acid (dissolved in the organic solvent) will cause deprotonation of benzoic acid (now negatively charged). The compound was in the organic layer, but after mixing it is in the aqueous layer. [Correct Answer] 
Adding strong acid to benzoic acid (dissolved in the organic solvent) will cause deprotonation of benzoic acid (now negatively charged). The compound was in the organic layer, but after mixing it is in the aqueous layer.

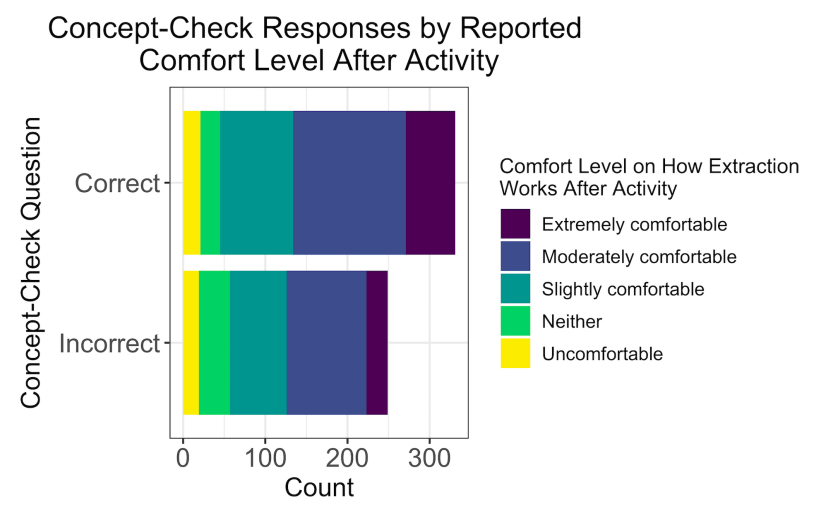

Figure 4. Students Who Answered Concept Questions Correctly or Incorrectly

\section{CONCLUSION}

The Extraction on Paper Activity allows students to walk through the thought processes required to conduct a liquid-liquid extraction in a lab, but in a lower stress setting. The Activity highlights principles of immiscibility, polarity, acid-base reactivity, and compound isolation in the context of an extraction. The Extraction on Paper Activity is amenable to demonstrating simple liquid-liquid extractions and acid-base extractions, and can be used with any compound mixture separation. The Activity can also be used in a variety of different learning settings, including small and large lectures, discussions, office hours, tutoring sessions, and labs. Students self-reported having an increased level of comfort in understanding how and why extraction works at the molecular level. An increased level of comfort in understanding extraction using this activity could lead to an increase in comfort level with performing an extraction in a laboratory setting. 


\section{ASSOCIATED CONTENT}

Supporting Information

Notes for instructors (PDF)

Worksheet with blanks (PDF)

Customizable worksheet with blanks (PDF)

Tabulated survey results (PDF)

\section{AUTHOR INFORMATION}

*E-mail: rlink@uci.edu

\section{ACKNOWLEDGMENTS}

We thank Dr. Kim Edwards, Dr. Amanda Holton, and Dr. Steve Mang for guidance and feedback on the worksheet's design. We also thank the members of Simon Lam's stockroom for helping assemble worksheets for use in lectures. We thank the chemistry peer tutors and Chem 51L students for feedback on the activity design.

\section{REFERENCES}

(1) Freeman, S.; Eddy, S. L.; McDonough, M.; Smith, M. K.; Okoroafor, N.; Jordt, H.; Wenderoth, M. P. Active Learning Increases Student Performance in Science, Engineering, and Mathematics. Proc. Natl. Acad. Sci. U. S. A. 2014, 111 (23), 8410-8415.

(2) Blank, E. W. Visual Demonstration of the Extraction Theory. J. Chem. Educ. 1935, 12 (4), 179.

(3) Kelly, T. R. A Simple, Colorful Demonstration of Solubility and Acid/base Extraction Using a Separatory Funnel. J. Chem. Educ. 1993, 70 (10), 848.

(4) Horowitz, G. A Discovery Approach to Three Organic Laboratory Techniques: Extraction, Recrystallization, and Distillation. J. Chem. Educ. 2003, 80 (9), 1039.

(5) Climent-Bellido, M. S.; Martínez-Jiménez, P.; Pontes-Pedrajas, A.; Polo, J. Learning in Chemistry with Virtual Laboratories. J. Chem. Educ. 2003, 80 (3), 346. 
(6) Turner, D. E. An Experiment to Demonstrate the Effect of $\mathrm{pH}$ on Partition Coefficients in Liquid-Liquid Extraction. J. Chem. Educ. 1994, 71 (2), 173.

(7) Raydo, M. L.; Church, M. S.; Taylor, Z. W.; Taylor, C. E.; Danowitz, A. M. A Guided Inquiry Liquid/Liquid Extractions Laboratory for Introductory Organic Chemistry. J. Chem. Educ. 2015, 92 (1), 139-142.

(8) Celius, T. C.; Peterson, R. C.; Anderson-Wile, A. M.; Kraweic-Thayer, M. From Observation to Prediction to Application: A Guided Exercise for Liquid-Liquid Extraction. J. Chem. Educ. 2018, 95 (9), 1626-1630.

(9) Hill, J. W.; McGurran, J. P. A Simple Model for Visualizing an Organic Extraction. J. Chem. Educ. 1990, 67 (4), 303. 